\title{
In vitro maturation impacts cumulus-oocyte complex metabolism and stress in cattle
}

\author{
Maite del Colladoํ, Juliano C da Silveira ${ }^{1}$, Marcelo L F Oliveira ${ }^{1}$, Bárbara M S M Alves ${ }^{1}$, \\ Rosineide C Simas², Adriana T Godoy², Mirela B Coelho², Lygia A Marques², \\ Mateus M Carriero', Marcelo F G Nogueira ${ }^{3}$, Marcos N Eberlin², Luciano A Silva ${ }^{1}$, \\ Flávio V Meirelles ${ }^{1}$ and Felipe Perecin ${ }^{1}$
}

\begin{abstract}
${ }^{1}$ Veterinary Medicine Department, Faculty of Animal Science and Food Engineering (FZEA), University of Sao Paulo (USP), Pirassununga, São Paulo, Brazil, ${ }^{2}$ ThoMSon Mass Spectrometry Laboratory, Institute of Chemistry, University of Campinas, Campinas, São Paulo, Brazil and ${ }^{3}$ Biological Sciences Department, School of Science, Humanities and Languages, Sao Paulo State University, Assis, São Paulo, Brazil
\end{abstract}

Correspondence should be addressed to F Perecin; Email: fperecin@usp.br

\begin{abstract}
The influence of in vitro maturation (IVM) in oocytes is still not totally understood. The aim of this study was to determine the influence of IVM on the metabolism and homeostasis of bovine cumulus-oocyte complexes. In the present study, we demonstrated that IVM leads to accumulation of neutral lipids associated with differential levels of the mono-, di- and triacylglycerols in both cumulus cells and oocytes. We observed that in vitro-matured oocytes exhibited decreased glutathione and reactive oxygen species levels and a lower ATP/ADP ratio when compared to in vivo-matured oocytes, with no significant differences in metabolism and stress-related mRNA or miRNA levels. Moreover, in addition to an increase in lipids in in vitro-matured cumulus cells, fatty acid synthesis and accumulation as well as glycolysis pathway genes were upregulated, whereas those affiliated with the $\beta$-oxidation pathway were decreased. Our gene expression data in cumulus cells suggest the disruption of endoplasmic reticulum stress, apoptosis and cellular stress response pathways during IVM. Furthermore, a total of 19 miRNAs were significantly altered by the maturation process in cumulus cells. These results indicate some new negative influences of the in vitro system in cumulus-oocyte complexes, demonstrating the occurrence of functional disruption in lipid metabolism and stress pathways and showing evidences suggesting the occurrence of altered mitochondrial activity and energy metabolism during IVM, with a massive dysregulation of the corresponding transcripts in the surrounding cumulus cells.

Reproduction (2017) $154881-893$
\end{abstract}

\section{Introduction}

Assisted reproduction techniques are widely used as a means to rescue fertility in several species and as a tool of genetic improvement in cattle. The in vitro system attempts to mimic the in vivo environment; however, there is a myriad of factors that can contribute to lower embryo production and quality. Although the culture environment is known to lead to stress and lipid accumulation after in vitro embryo production, impairing embryo cryotolerance and pregnancy rates in bovines (Goto et al. 1993, Wright \& Ellington 1995, Abe et al. 2002, Rizos et al. 2002, Seidel 2006), it is unknown whether these effects are the consequence of exposure of the oocyte to the in vitro environment or if other underlying mechanisms may be involved. Therefore, it is important to perform comprehensive studies regarding these contributing factors during oocyte in vitro maturation (IVM) for comparison with the in vivo-produced counterparts.

In this context, oocyte maturation is dependent on an orchestrated sequence of events involving oocyte and follicular constituents, such as cumulus cells, to produce oocytes that are competent to support development. Thus, studies considering cumulus-oocyte complexes (COCs) as interdependent compartments and focusing on their behavior during maturation are necessary to understand the influence of IVM on cell metabolism and homeostasis.

Oxidative phosphorylation constitutes the most important energy pathway during oocyte maturation. Given that oocytes and embryos are not able to perform glycolysis prior to the compaction and blastulation stages, the cumulus cells are responsible for providing pyruvate and lactate to the oocyte (Khurana \& Niemann 2000, Thompson et al. 2015). However, $\beta$-oxidation, another metabolism pathway, has also been recognized as being 
highly important during oocyte maturation. Studies have shown an increase of oocyte quality and embryo development rate when $\beta$-oxidation was increased during IVM, suggesting the occurrence of an energy imbalance during IVM and pointing to deficient $\beta$-oxidation as being likely responsible (Downs et al. 2009, Dunning et al. 2010, 2014, Paczkowski et al. 2013). Moreover, it was demonstrated that IVM leads to an increase in lipid accumulation that does not occur during in vivo maturation (Del Collado et al. 2016), suggesting an alteration in lipid metabolism during in vitro culture.

Furthermore, the properties of energy metabolism and its association with cellular stress responses during oocyte maturation also remain poorly understood. Although most studies consider metabolism and homeostasis modifications as independent effects during the in vitro process, some have demonstrated a correlation between increased lipids and cellular stress (Borradaile et al. 2006, Diakogiannaki et al. 2008).

Recently, the roles of certain miRNAs in maturation have been described, the majority of which are expressed in cumulus cells, although several effects were noted on oocyte maturation and embryo development as well (Hossain et al. 2012, Miles et al. 2012, Shi et al. 2015). In addition, the miRNA-mediated regulation of metabolism and stress-related genes has been demonstrated in several species (Duru et al. 2015, Yang et al. 2015, Magenta et al. 2016); however, it is still unknown how miRNAs modulate these genes during oocyte maturation in bovines.

Therefore, the aim of this study was to determine the mechanisms influencing cumulus cell and oocyte metabolism and homeostasis during IVM by analyzing energy substrates and metabolites as well as stressrelated compounds, in addition to transcripts and posttranscriptional regulators of energy metabolism, ER stress, apoptosis and cellular responses to stress. Wehypothesized that the in vitro environment causes metabolic disruption, increasing lipid accumulation, altering the mono-, diand triacylglycerols profile, leading to cellular stress of the COC. In order to address these questions, we performed several measurements in oocytes, indicative of potential metabolic and stress-related modifications acquired during IVM. As we did not observe alterations in transcriptional or post-transcriptional regulators in oocytes that could explain the effects of the in vitro system, we investigated the mechanisms leading to the noted alterations in cumulus cells.

\section{Materials and methods}

Reagents were purchased from Sigma Chemical Co. unless otherwise indicated. The study was approved by the Bioethical Committee of the FZEA - University of Sao Paulo, Pirassununga, SP, Brazil, under protocol number 14.1.675.74.7. Experiments were conducted in accordance with International Guiding Principles for Biomedical Research
Involving Animals, meeting the Society for the Study of Reproduction standards.

\section{Immature and in vitro-matured oocyte collection}

We collected grade I and II COCs by postmortem follicular aspiration of 3-8 $\mathrm{mm}$ follicles from Nellore (Bos indicus) ovaries obtained from slaughterhouse carcasses to obtain immature and in vitro-matured oocytes. The denuded oocytes and corresponding cumulus cells were recovered from immature COCs and stored for further analysis. A fraction of the immature COCs was assigned for IVM at $38.5^{\circ} \mathrm{C}$ and $5 \% \mathrm{CO}_{2}$ in air and high humidity for $24 \mathrm{~h}$. The IVM media was composed of TCM-199 with Earle's Salts, L-glutamine and $2.2 \mathrm{~g} / \mathrm{L}$ sodium bicarbonate (GIBCO BRL), $8.75 \times 10^{-4} \mathrm{IU} / \mathrm{mL}(0.5 \mu \mathrm{g} / \mathrm{mL})$ follicle-stimulating hormone ( $\mathrm{FSH}$; Ourofino Saude Animal, Cravinhos, Brazil), $50 \mu \mathrm{g} / \mathrm{mL}$ human chorionic gonadotropin (hCG; Vetecor; Ourofino Saude Animal), $50 \mu \mathrm{g} / \mathrm{mL}$ gentamicin, $0.2 \mathrm{mM}$ sodium pyruvate and $10 \%$ fetal bovine serum (FBS, GIBCO). After maturation, cumulus cells from expanded COCs were separated from the oocyte with needles. The oocytes were totally denuded by continuous pipetting with 135- $\mu \mathrm{m}$-diameter stripper tips. All oocytes were evaluated for nuclear maturation and only MII oocytes presenting the first polar body (1 PB) and the corresponding cumulus cells were used for further analysis. Samples assigned for RNA were immediately placed in liquid nitrogen and stored at $-80^{\circ} \mathrm{C}$ until further use. The samples from 12 different IVM routines were obtained simultaneously with in vivo oocyte collections.

\section{In vivo-matured oocyte collection}

To obtain in vivo-matured oocytes, the ovarian follicular wave was synchronized in 33 cyclic Nellore cows and animals were submitted to follicular superstimulation during 12 different ovum pick-up (OPU) sessions. On the first day (Day 0), females received $0.5 \mathrm{mg}$ cloprostenol (Sincrocio, OuroFino Saude Animal) and follicular wave synchronization was induced by follicular ablation associated with the administration of $2 \mathrm{mg}$ benzoate estradiol (Sincrodiol, Ourofino Saude Animal) and intravaginal progesterone-releasing device (IPRD) implants (Sincrogest, OuroFino Saude Animal). Follicular superstimulation was initiated on Day 4. A total of $133 \mathrm{mg}$ of FSH (Folltropin, Bioniche Animal Health Canada Inc., Belleville, ON, Canada) per cow was divided into 8 decreasing doses administered every $12 \mathrm{~h}$. Along with the sixth dose of FSH, $0.5 \mathrm{mg}$ of cloprostenol was administered and $12 \mathrm{~h}$ later, the IPRD was removed. On Day 8, females received $0.02 \mathrm{mg}$ buserelin acetate (GnRH analog, Sincroforte, OuroFino Saude Animal) and 25-26h later expanded COCs were recovered using the OPU method. In vivo-matured oocytes were evaluated for nuclear maturation and selected based on 1 PB extrusion and only matured oocytes (identified as MII oocytes) and corresponding cumulus cells were used for further analysis.

\section{High-performance liquid chromatography mass spectrometry analysis (HPLC-MS)}

HPLC-MS was used to identify lipids in immature, in vivo- and in vitro-matured oocytes and cumulus cells (Supplementary 
Fig. 1, see section on Supplementary Data given at the end of this article). For each group, five pools of cumulus cells retrieved from seven COCs each or five pools of ten denuded oocytes were used.

\section{Chemicals and reagents}

Methanol (MeOH, HPLC grade), chloroform $\left(\mathrm{CHCl}_{3}, \mathrm{HPLC}\right)$, acetonitrile (ACN, HPLC), water $\left(\mathrm{H}_{2} \mathrm{O}\right.$, ultra-gradient grade) and isopropanol (IPA, HPLC) were purchased from JT Baker (Avantor, Center Valley, PA, USA). MS reference standard $d_{5^{-}}$ Triacyglycerol Internal Standard mixture and the reference standard $\mathrm{d}_{5}$-Diacylglycerol Internal Standard mixture were from Avanti Polar Lipids, Alabaster, AL, USA. Strata silica columns $(100 \mathrm{mg}$ for $1 \mathrm{~mL}$ ) were from Phenomenex (Torrance, CA, USA).

\section{Preparation of standard internal solutions}

Reference substances were combined at $200 \mathrm{ng} / \mathrm{mL}$ each in $\mathrm{CHCl}_{3}: \mathrm{MeOH}(1: 1, \mathrm{v} / \mathrm{v})$ and diluted to $40 \mathrm{ng} / \mathrm{mL}$ in $\mathrm{ACN}$ as a working solution.

\section{Extraction of sample lipids}

For each pool $(10 \mu \mathrm{L}$ in PBS buffer), $150 \mu \mathrm{L}$ of water, $450 \mu \mathrm{L}$ IPA and $50 \mu \mathrm{L}$ internal standard mix $(40 \mathrm{ng} / \mathrm{mL})$ was added and vortexed. Then, the solution was sonicated (20 min), centrifuged $(15,000 \mathrm{~g}$ for $5 \mathrm{~min}$ at room temperature) and the supernatant was collected and dried under a constant stream of nitrogen $\left(45^{\circ} \mathrm{C}\right)$.

Light petroleum-diethyl ether ( $2 \mathrm{~mL}, 90: 10$, v/v) was added to the dried extract, which was cleaned-up with an SPE column (100 mg), previously conditioned with $2 \mathrm{~mL}$ of water and $5 \mathrm{~mL}$ of light petroleum-diethyl ether (90:10, v/v). The clean-up with the SPE column is important to remove phospholipids that could interfere with mono-, di- and triacylglycerol (MG, DG, and TG, respectively) measurements. Lipids were eluted with $5 \mathrm{~mL}$ diethyl ether containing MG, DG and TG (MárquezRuiz et al. 1996) and evaporated under a constant stream of nitrogen $\left(45^{\circ} \mathrm{C}\right)$. Lipid extracts were diluted with $50 \mu \mathrm{L}$ ACN:IPA: $\mathrm{H}_{2} \mathrm{O}$ (79:5:1 - $5 \mathrm{mM}$ ammonium acetate), vortexed (3 min), filtered with polyvinylidene fluoride membrane filters $(0.22-\mu \mathrm{m}$ pores $)$ for $5 \mathrm{~min}(10,000 \mathrm{~g})$ and injected to the HPLC. The identified lipids were quantified against their corresponding internal standard (for MG, TG was used as the internal standard).

\section{Analysis}

Lipids were quantified using a 5500 Q-TRAP mass spectrometer (Sciex, Framingham, MA, USA) with an ESI interface in positive mode and a 1260 series HPLC Agilent device (Agilent). Data acquisition and analyses were performed using Analyst software (version 1.6.1; Sciex). Labeled and unlabeled lipids were separated on a Phenomenex C8 column $(3.0 \times 150 \mathrm{~mm}$, $2.7 \mu \mathrm{m}$, Phenomenex) at a flow rate of $300 \mu \mathrm{L} / \mathrm{min}$. Mobile phase B (ACN:IPA 80:20, v/v) was linearly increased from 1 to $99 \%$ over $5 \mathrm{~min}$, kept constant for $20 \mathrm{~min}$, returned to the initial condition over $1 \mathrm{~min}$ and kept constant for an additional 5 min. Mobile phase A consisted of ACN:5 mM ammonium acetate 1:1. Injection volume was $5 \mu \mathrm{L}$. The optimized ESI interface parameters were the following: capillary voltage, $5 \mathrm{kV}$; curtain gas 10 psi; nebulizer gas 45 psi; desolvation gas 20 psi; CAD gas $12 \mathrm{psi}$; source temperature, $450^{\circ} \mathrm{C}$. Supplementary Table 1 for optimized multiple reaction monitoring (MRM) parameters such as declustering potential, collision energies and collision cell exit potential for each lipid (synthetic lipidlabeled solutions).

\section{Quantification of reduced glutathione (GSH), reactive oxygen species (ROS), ATP and ADP levels in immature and in vivo- and in vitro-matured oocytes}

For ROS and GSH quantification, a total of 37 (immature), 29 (in vivo-matured) or 24 (in vitro-matured) oocytes were stained with $5 \mu \mathrm{M}$ CellRoX Green (Molecular Probes) and $10 \mu \mathrm{M}$ Celltracker Blue CMF2 HC (Molecular Probes) and fixed according to the manufacturer's instructions. Then, the oocytes were analyzed on an LSM 710 confocal microscope (Carl Zeiss) using a $63 \times$ objective in oil and an Argon laser $(488 \mathrm{~nm})$ and diode $(405 \mathrm{~nm})$, with the excitation/emission adjusted for each probe parameter. All images were captured under the same parameters, using sequential acquisition. We captured three images of each oocyte; these were assessed using ImageJ software (NIH; http://rsb.info.nih.gov/ij/) to measure the fluorescence intensity (f.i.) of GSH and ROS.

ATP and ADP content and the ATP/ADP ratio of 30 individual oocytes per group in five replicates were measured using the bioluminescence ApoSENSOR ATP Assay Kit (BioVision) according to the manufacturer's instructions. Briefly, we measured the ATP absorbance by a luminescence analysis. Then, the samples underwent an enzymatic reaction to convert the ADP to ATP, and the ATP absorbance was measured again. The levels of ADP were calculated from the difference in absorbance values obtained in the first and second measurements. A five-point ATP standard curve $(0.15,0.075,0.0375,0.01875$ and $0.009375 \mu \mathrm{M})$ was used to estimate sample concentration.

\section{RNA extraction and messenger RNA and microRNA reverse transcription}

For total RNA extraction, we used five pools of 20 immature and in vivo- or in vitro-matured oocytes and five or eight different cumulus cell pools retrieved from 20 immature or 10 in vivo- and in vitro-matured COCs. Total RNA extraction was performed using TRIzol reagent and miRNAse kit (Qiagen). Reverse transcription of mRNA was performed using the HighCapacity cDNA Reverse Transcription kit (Applied Biosystems); for miRNA reverse transcription, we utilized the miScript polymerase chain reaction (PCR) system (Qiagen \#218193) according to manufacturer instruction.

\section{Real-time PCR analysis of genes related to lipid metabolism and to cellular stress in immature, in vivo- and in vitro-matured oocytes and cumulus cells}

For mRNA expression, we performed microfluidic transcript level analysis of 25 cellular metabolism and stress-related 
genes (Supplementary Table 2) utilizing the Biomark HD System (Fluidigm, South San Francisco, CA, USA). For this analysis, we used PPIA, GAPDH and ACTB as endogenous performed using Bos taurus-specific TaqMan assays (20x, Applied Biosystems). After the pre-amplification process using TaqMan PreAmp Master Mix (Applied Biosystems), samples were diluted 5 -fold and analyzed by reverse transcriptionquantitative PCR (RT-qPCR) using the TaqMan Universal PCR Master Mix (2×, Applied Biosystems) and TaqMan assays (20×, Applied Biosystems) in the 96.96 Dynamic Array Integrated Fluidic Circuits on the Biomark HD System (Fluidigm) using the protocol TaqMan GE $96 \times 96$ Standard, according to manufacturer's instructions.

To complement this work, we also performed realtime RT-qPCR of several genes related to lipid synthesis and accumulation and transport (PLIN2, PLIN3 and SREBP1), $\beta$-oxidation (CPT1A, CPT1B and CPT2) and endoplasmic reticulum (ER) stress (CHOP10, IRE, PERK, and ATF6), where PPIA, SDHA and YWAHZ were used for the endogenous control genes. Primers were designed based on bovine sequences available at GenBank (Supplementary Table 3); these were tested for efficiency and the obtained amplicons were sequenced to ensure their specificity. Real-time RT-qPCR reactions were performed using $10 \mathrm{ng}$ cDNA for each gene and $75 \mathrm{nM}$ of each primer with the Power SYBR Green PCR Master Mix kit (Applied Biosystems) in the QuantStudio 6 Flex PCR System (Applied Biosystems).

\section{Real-time PCR expression analysis of miRNAs predicted to regulate genes involved in cellular metabolism and stress in immature, in vivo- and in vitro-matured oocytes and cumulus cells}

To evaluate whether miRNAs predicted to regulate the studied genes were altered following IVM, we selected miRNAs identified using bioinformatics. The relative levels of 88 bovine mature miRNAs (Table 1 and Supplementary Table 4) were examined in immature, in vivo- and in vitromatured oocytes and cumulus cells. Specifically, the miRNAs evaluated in this study were chosen based on validated or predicted interaction with the target genes. We used three for bovine genes using miRTarBase (http://mirtarbase.mbc. nctu.edu.tw/) were directly included in this study. Next, those without validation for bovine genes were compared with their homologs validated for humans by analyzing the similarity between the human and bovine miRNAs using miRBase (http:// mirbase.com, Supplementary Table 5) and their binding site on the target gene as predicted by TargetScan software (www. targetscan.otg). Finally, if the target gene had no validated miRNA, we chose bovine miRNAs predicted to target the genes using TargetScan.

The RT-qPCR reaction was performed using Quantitec SYBR Green (Qiagen), $10 \mu \mathrm{M}$ Universal reverse primer (Qiagen), $10 \mu \mathrm{M}$ miRNA-specific forward primer (Supplementary Table 6), and 60 pg cDNA and analyzed using the QuantStudio 6 Flex PCR System. The Ct values were normalized with the geometric mean control genes. Microfluidic gene expression analysis was criteria for miRNA inclusion. First, miRNAs already validated

Table 1 Bovine miRNA amplified in this study and their respective target genes.

\begin{tabular}{|c|c|}
\hline miRNAs & Target genes \\
\hline bta-let-7a-5p & CASP3 \\
\hline bta-miR-15a & $A C S L 6, B C L 2$ \\
\hline bta-miR-15b & FASN, ACSL6, BCL2 \\
\hline bta-miR-16a & $A C S L 6, B C L 2$ \\
\hline bta-miR-16b & FASN, ACSL6, BCL2 \\
\hline bta-miR-17-5p & PFKP, CASP9 \\
\hline bta-miR-20a & PFKP, CASP9 \\
\hline bta-miR-20b & PFKP, CASP9 \\
\hline bta-miR-23a & ACSL3 \\
\hline bta-miR-23b-3p & ACSL3 \\
\hline bta-miR-24-3p & ACSL6 \\
\hline bta-miR-25 & ATF6 \\
\hline bta-miR-26a & ACSL3 \\
\hline bta-miR-26b & ACSL3 \\
\hline bta-miR-27a-3p & NRF2 \\
\hline bta-miR-29b & $B A X$ \\
\hline bta-miR-29c & $B A X$ \\
\hline bta-miR-29d-3p & $B A X$ \\
\hline bta-miR-30a-5p & GRP78 \\
\hline bta-miR-30b-5p & CAT \\
\hline bta-miR-30c & CASP3 \\
\hline bta-miR-30d & CASP3 \\
\hline bta-miR-32 & ATF6 \\
\hline bta-miR-33a & SREBP1, CPT1A \\
\hline bta-miR-33b & CPT1A \\
\hline bta-miR-34a & BCL2 \\
\hline bta-miR-34b & $B C L 2$ \\
\hline bta-miR-34c & $B C L 2$ \\
\hline bta-miR-92a & ATF6 \\
\hline bta-miR-92b & ATF6 \\
\hline bta-miR-93 & PFKP, CASP9 \\
\hline bta-miR-96 & CHOP10 \\
\hline bta-miR-106a & PFKP, CASP9 \\
\hline bta-miR-106b & PFKP, CASP9 \\
\hline bta-miR-124a & CPT1A \\
\hline bta-miR-124b & CPT1A \\
\hline bta-miR-125a & ATF6 \\
\hline bta-miR-125b & $B C L 2$ \\
\hline bta-miR-132 & ACSL3 \\
\hline bta-miR-136 & SOD2 \\
\hline bta-miR-137 & ACSL6 \\
\hline bta-miR-142-5p & ACSL6, CASP9, NRF2 \\
\hline bta-miR-143 & $P G K 1, B C L 2$ \\
\hline bta-miR-144 & $N R F 2$ \\
\hline bta-miR-146a & PGK1 \\
\hline bta-miR-146b & PGK1 \\
\hline bta-miR-149-5p & ATF6 \\
\hline bta-miR-153 & $N R F 2$ \\
\hline bta-miR-181a & $S C D, A C S L 6, C P T 1 A, B C L 2$ \\
\hline bta-miR-181b & ACSL6, CPT1A, BCL2, GRP78 \\
\hline bta-miR-181c & ACSL6, CPT1A, BCL2 \\
\hline bta-miR-181d & ACSL6, CPT1A, BCL2 \\
\hline bta-miR-182 & $B C L 2$ \\
\hline bta-miR-186 & ACSL6, CASP6 \\
\hline bta-miR-195 & ACSL6, BCL2 \\
\hline bta-miR-199a-3p & ATF6 \\
\hline bta-miR-199a-5p & IRE1, ATF6, GRP78 \\
\hline bta-miR-200b & ACSL3 \\
\hline bta-miR-200c & ACSL3 \\
\hline bta-miR-202 & $A C S L 3$ \\
\hline bta-miR-204 & ELOVL6, BCL2 \\
\hline bta-miR-206 & G6PD \\
\hline
\end{tabular}




\begin{tabular}{ll} 
bta-miR-211 & ELOVL6, BCL2 \\
bta-miR-218 & ACSL6 \\
bta-miR-223 & ACSL3 \\
bta-miR-302b & PFKP \\
bta-miR-302c & PFKP \\
bta-miR-302d & PFKP \\
bta-miR-330 & ACSL6, SOD2 \\
bta-miR-346 & CASP9 \\
bta-miR-365-3p & PGK1, BCL2 \\
bta-miR-374a & ACSL6 \\
bta-miR-374b & ACSL6 \\
bta-miR-378 & ATF6 \\
bta-miR-381 & ACSL3 \\
bta-miR-421 & ACSL3 \\
bta-miR-424-5p & ACSL6 \\
bta-miR-429 & ACSL3, CPT2 \\
bta-miR-448 & ACSL6 \\
bta-miR-449a & BLC2 \\
bta-miR-495 & ACSL6, PGK1 \\
bta-miR-497 & ACSL6, BCL2 \\
bta-miR-499 & $C P T 1 A$ \\
bta-miR-503-5p & ACSL6, ATF6 \\
bta-miR-582 & ACSL6 \\
bta-miR-670 & ATF6 \\
bta-miR-873 & PGK1, GPX1 \\
bta-miR-875 & ATF6 \\
\hline
\end{tabular}

of RNU43 snoRNA, Hm/Ms/Rt U1 snRNA and bta-miR-99b. The expression value was calculated using the method of $2^{-\Delta C t}$.

\section{Statistical analysis}

The lipid accumulation and the ATP and ADP data were compared using the non-parametric Kruskal-Wallis test. Data of GSH and ROS quantification along with MG, DG and TG quantitation as well as the mRNA and miRNA expression data were subjected to analysis of variance (ANOVA) and the averages were compared using the Tukey test. The equality of the variances was tested using the Levene test and normality was assessed with the Shapiro-Wilk test. All analyses were performed using SAS software (SAS Institute Inc., Cary, NC, USA) and a level of significance of $5 \%$ was considered for all tests.

\section{Results}

\section{In vitro culture of COCs increased DGs and TGs in oocytes}

No difference was observed in the total amount of MGs in oocytes after IVM when compared to the in vivo system (Fig. 1A). However, when analyzing the individual profiles of MGs, we noticed a general increase of long-chain fatty acids in in vitro vs in vivo oocytes (Supplementary Fig. 2A). In particular, seven MGs increased during both in vivo and in vitro maturation (12:0, 14:0, 16:0, 18:2, 18:3, 24:0 and 26:1; Supplementary Fig. 2A). Of these, only MG 26:1 was increased in in vivo-matured oocytes compared to in vitro, whereas MGs 12:0, 16:0 18:1, 18:3, 20:4 and 24:0 showed higher levels in IVM compared to in vivo-matured oocytes (Supplementary Fig. 2A). In addition, five MGs were increased solely after IVM (14:1, 16:1, 18:0, 18:1 and 20:0), whereas MG18:1 was decreased after in vivo maturation when compared to immature oocytes.

In comparison, the total levels of DGs and TGs increased only in the IVM-derived oocytes (Fig. 1B and $\mathrm{C})$. We identified eight DGs that increased during both maturation systems $\left((16: 0)_{2}, \quad(17: 0)_{2}, \quad(19: 0)_{2}\right.$, $(20: 2)_{2},(20: 5)_{2},(24: 0)_{2},(24: 1)_{2}$ and $\left.(24: 4)_{2}\right)$ and five that increased only in IVM $\left((18: 0)_{2},(18: 2)_{2},(18: 3)_{2},(20: 4)_{2}\right.$ and $\left.(22: 4)_{2}\right)$ (Supplementary Fig. 2B). Among those that increased during both maturation systems, four DGs showed a preferential increase in the in vitro compared to the in vivo group $\left((16: 0)_{2},(24: 0)_{2},(24: 1)_{2}\right.$, and $\left.(24: 4)_{2}\right)$. Additionally, with the exception of $(17: 0)_{2} \quad 17: 1$, which exhibited no difference between the in vivo and in vitro groups, all the other eight TGs species quantified in this study $\left((14: 0)_{2} \quad 16: 1,(15: 0)_{2} \quad 18: 1,(16: 0)_{2} \quad 18: 0,(16: 2)_{2}\right.$ 18:1, (20:0) $)_{2} 20: 1,(20: 2)_{2}$ 18:2, (20:4) $)_{2} 18: 2$ and (20:5) 22:6) were higher in in vitro-matured oocytes compared to their in vivo-matured counterparts (Supplementary Fig. 2C).

\section{IVM decreases GSH levels and ATP/ADP ratio in oocytes compared to in vivo maturation}

GSH levels increased in both maturation processes compared to immature oocytes $(15.43 \pm 7.45$ f.i.), although we observed relatively higher levels in in vivo- $(30.49 \pm 13.80$ f.i.) compared to in vitro-matured oocytes (22.84 \pm 8.90 f.i.) (Fig. 2A). Furthermore, ROS levels between immature $(11.39 \pm 4.30$ f.i. $)$ and matured groups did not differ (Fig. 2B). However, ROS levels were higher in the in vivo $(13.53 \pm 4.54$ f.i.) compared with the in vitro $(10.72 \pm 3.26$ f.i.) group (Fig. $2 \mathrm{~B})$.

We also observed increased ATP levels in the in vitro $(1.784 \pm 0.333 \mathrm{pmol} /$ oocyte $)$ compared to the

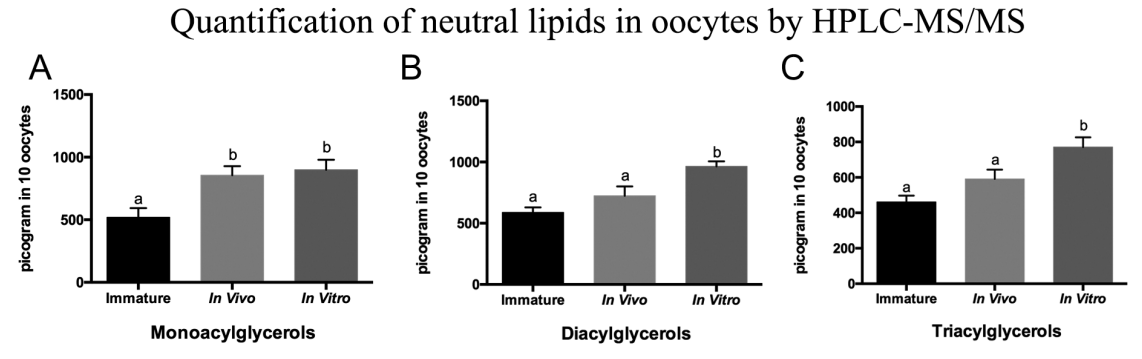

Figure 1 Quantitation of neutral lipids in oocytes by HPLC-MS/MS. Absolute quantities of monoacylglycerols (A), diacylglycerols (B), and triacylglycerols $(\mathrm{C})$ in oocytes from immature, in vivo-matured, and in vitromatured COCs. Different letters above the bars within a graph indicate significant differences $(P<0.05)$. Bars depict the means and error bars depict S.E.M. 


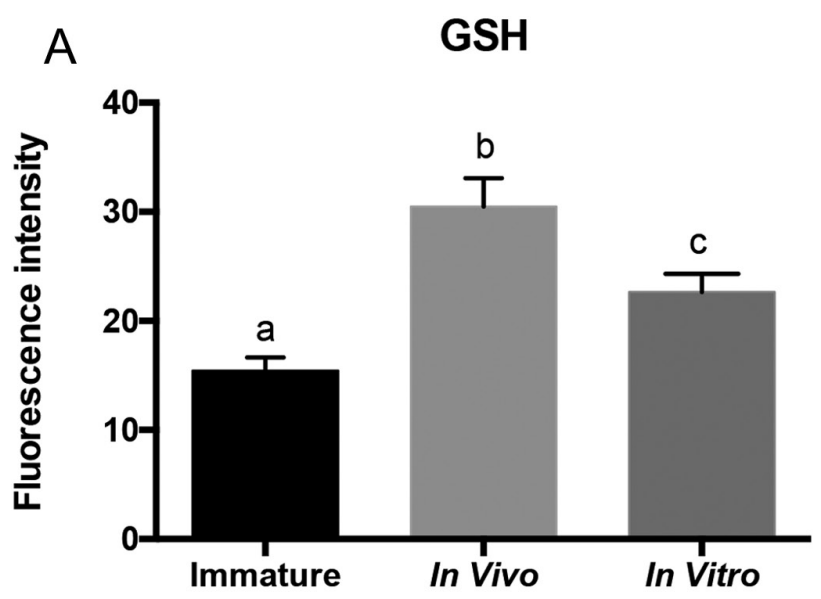

B

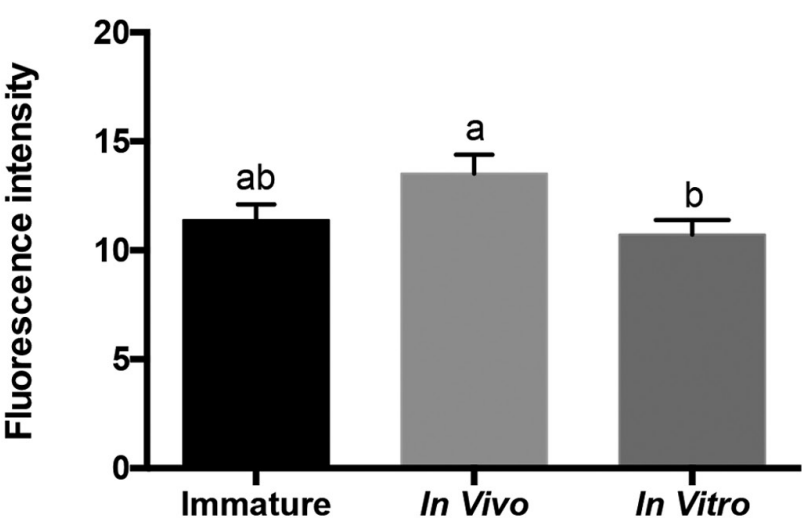

Figure $2 \mathrm{GSH}$ (A) and ROS (B) quantification before (immature) and after in vivo and in vitro maturation in oocytes. Different letters above the bars for the same graph indicate significant differences $(P<0.05)$. Bars depict the means and error bars depict s.E.M.

in vivo $(1.551 \pm 0.420 \mathrm{pmol} /$ oocyte $)$ and immature $(1.437 \pm 0.672 \mathrm{pmol} /$ oocyte $)$ groups. Additionally, we observed decreased ADP levels in in vivo oocytes (0.127 $\pm 0.126 \mathrm{pmol} / \mathrm{oocyte})$ compared to in vitro $(0.235 \pm 0.165 \mathrm{pmol} / \mathrm{oocyte})$ and immature $(0.176 \pm 0.110 \mathrm{pmol} /$ oocyte $)$ oocytes $(P=0.06$; Fig. 3A and $\mathrm{B}$ respectively for ATP and ADP). Furthermore, to evaluate the energy demand, we calculated the ATP/ ADP ratio. The in vivo group $(36.88 \pm 37.34)$ showed an increased energy demand when compared to in vitro $(12.87 \pm 12.53)$ and immature $(12.74 \pm 12.73)$ groups (Fig. 3C).
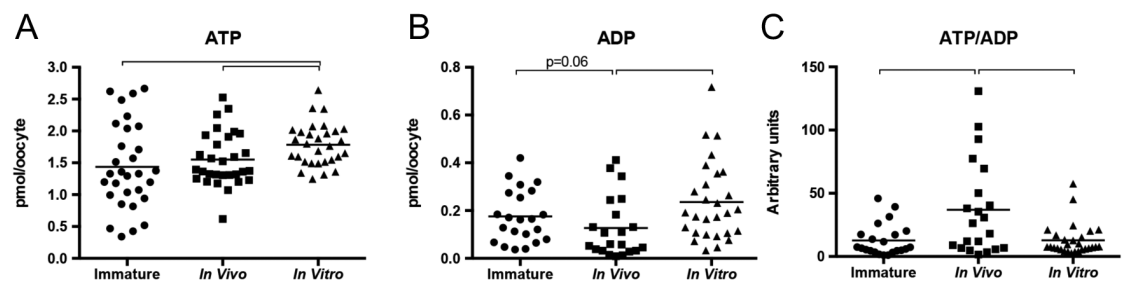

Figure 3 Scattered dot plots for ATP (A), ADP $(B)$, and ATP/ADP $(C)$ ratio quantities in immature and in in vivo- and in vitro-matured oocytes. Each dot represents an oocyte, lines indicate the means, and brackets indicate statistical difference $(P<0.05$; unless differently stated) between groups. 


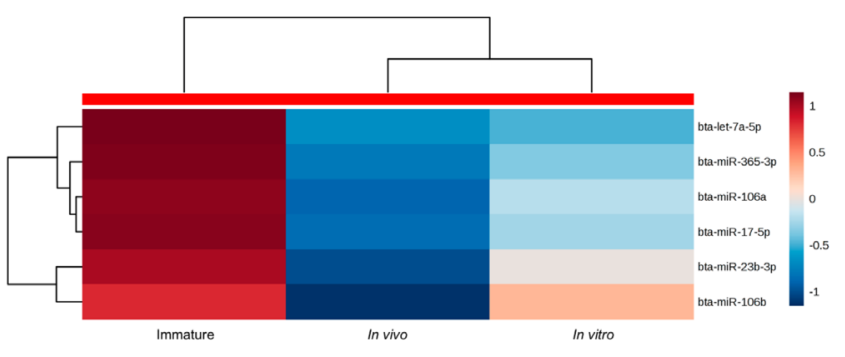

Figure 4 Hierarchical clustering and heatmap depicting expression levels of miRNAs differentially expressed among immature, in vivo-matured, and in vitro-matured oocytes.

immature cumulus cells; however, this pattern was not observed for four TG species ((16:0) $)_{2}$ 18:0, $(16: 2)_{2}$ 18:1, $(20: 2)_{2}$ 18:2 and $(20: 4)_{2}$ 18:2) between immature and in vivo groups (Supplementary Fig. 2F).

Total DG levels were also higher in cumulus cells obtained after IVM compared to immature or in vivo controls. These data, as well as the levels of DG species are provided in Fig. 5C and Supplementary Fig. 2E.

\section{Cellular metabolism and stress-related genes are altered in in vitro-matured cumulus cells}

The relative levels of cellular metabolism-related genes in cumulus cells differed for 27 out of 34 genes during maturation (Fig. 6 and Supplementary Table 7); additionally, a total of sixteen genes showed differences between in vivo and in vitro groups (Fig. 6). The metabolism-related genes ACACA, PLIN2, SCD, ACSL3, CPT1A, CPT2, G6PD and PKG1 presented increased levels during maturation regardless of the system when compared to the immature group (Fig. 6). Of these, ACACA, PLIN2, SCD, G6PD and PKG1 transcripts exhibited relatively higher levels in IVM. However, CPT1A demonstrated higher levels in in vivo, when compared to cumulus cells from in vitro-matured COCs. Conversely, SREBP1, FASN and ELOVL6 only exhibited decreased expression levels during in vivo maturation (Fig. 6), whereas SREBP1, ACSL6, CPT1B and PFKP transcript levels were increased only during IVM.

ER stress and apoptosis-related genes such as ATF6, $B A X, B C L 2$ and $C A S P 9$ were increased during maturation, whereas CHOP10 and GRP78 were decreased in both maturation groups (Fig. 6). Of these, BAX and BCL2 expression was relatively higher in cumulus cells from the in vitro compared with the in vivo group. Additionally, CASP3 levels were increased only during IVM, whereas IRE1, PERK and ATF4 transcripts maintained stable levels during maturation. The ER stress response and antioxidant-related genes NRF2, KEAP1, SOD1, GPX4, PRDX1 and CAT patterns also changed during maturation. NRF2 as well as the gene for its inhibitor, $K E A P 1$, increased during both maturation systems, although the in vivo group demonstrated relatively higher NRF2 transcript levels when compared to the IVM group. Conversely, GPX4 transcript levels decreased regardless of the maturation system. Moreover, SOD and CAT1 were increased in in vitro-matured cumulus cell compared to immature, while PRDX1 was higher in in vivo-matured cumulus cell compared to immature and in vitro-matured cumulus cells.

\section{MicroRNA levels are altered in cumulus cells after maturation in vitro and in vivo}

A total of 19 miRNAs varied owing to the maturation process (Fig. 7). Of these, eight miRNAs (miR-181b, miR-181d, miR-182, miR-204, miR-218, miR-27a-3p, miR-29d-5 and bta-mir-96) significantly increased and two miRNAs (miR-23a and miR-23b-3p) significantly decreased in cumulus cells following IVM compared to those from immature follicles. In comparison, 11 miRNAs (miR-181d, miR-182, miR-186, miR-199a-3p, miR-200c, miR-223, miR-27a-3p, miR-29c, miR29d-5p, miR-424-5p and miR-96) were significantly increased and three miRNAs (miR-17-5p, miR-23b-3p, and miR-330) were significantly decreased in cumulus cells after in vivo maturation compared to those from immature follicles. Notably, two miRNAs (miR-218 and miR-330) were significantly increased and six miRNAs (miR-182, miR-199a-3p, miR-200c, miR-223, miR$23 a$ and miR-424-5p) were significantly decreased in cumulus cells after in vitro compared to in vivo maturation. The expression values of all tested miRNAs from all cell groups are listed in Supplementary Table 8.

\section{Discussion}

Despite all efforts to understand how the in vitro system influences oocyte and embryo development, limitations
A

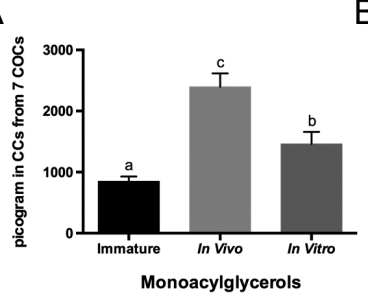

B

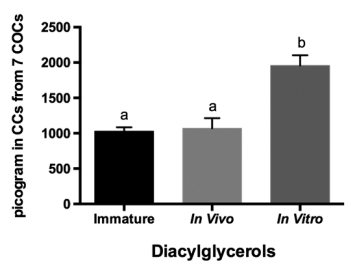

C

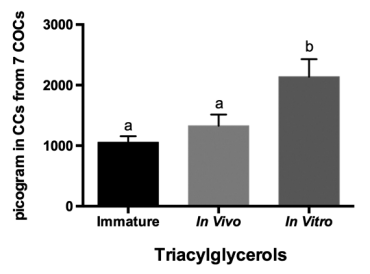

Figure 5 Quantitation of neutral lipids in cumulus cells (CCs) by HPLC-MS/MS. Absolute quantities of monoacylglycerols (A), diacylglycerols (B), and triacylglycerols $(\mathrm{C})$ in cumulus cells from immature, in vivo-matured, and in vitro-matured COCs. Different letters above the bars within a graph indicate significant differences $(P<0.05$, except for letters ' $a$ ' and ' $b$ ' in $A$, which indicate $P=0.06$ ). Bars depict the means and error bars depict S.E.M. 

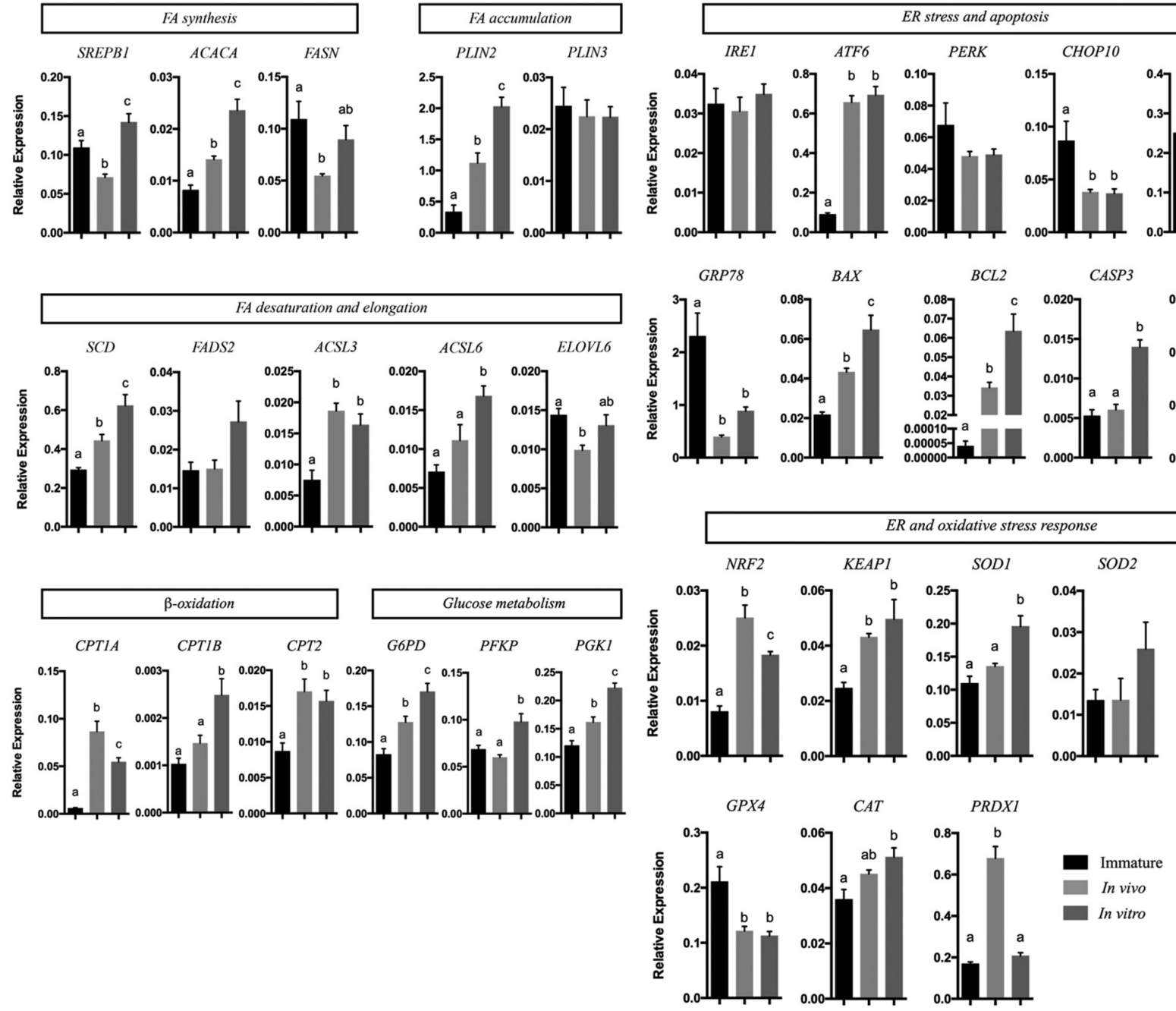

Figure 6 Expression of cellular metabolism and stress-related genes in cumulus cells from immature and in vivo- and in vitro-matured COCs. Different letters above the bars for the same gene indicate significant differences $(P<0.05)$. Bars depict the means and error bars depict S.E.M.

remain that render IVM far from equivalent to the in vivo conditions. Nevertheless, the means by which the oocyte maturation environment might influence these features are not clearly understood. Accordingly, in the current study, we addressed how the in vitro system influences metabolism and cellular homeostasis during oocyte maturation.

In particular, although some studies have described a decrease in lipid quantity in oocytes during IVM (Ferguson \& Leese 1999, Kim et al. 2001), we demonstrated by MS analysis that, unlike in vivo maturation, IVM leads to increased DGs and TGs in oocytes and cumulus cells. This result is in accordance to other studies that demonstrated higher lipid accumulation in oocytes after IVM, even without serum supplementation (Aardema et al. 2011, Del Collado et al. 2016). In addition, we demonstrated that IVM could modify MG, DG and TG patterns in COCs. Notably, different fatty acids may be helpful or harmful during maturation (McKeegan \& Sturmey 2011), indicating a possible influence of some fatty acid on oocyte quality during IVM. For example, palmitic acid (16:0) and linolenic acid (18:3) were showed to have detrimental effects in oocyte maturation and embryo development upon their appearance in high concentration during IVM (Leroy et al. 2005, Marei et al. 2009). As in our results, both fatty acids increased in oocytes in in vitro-matured compared with in vivo-matured groups, which indicates higher levels than what would be expected in physiological conditions. Detrimental effects owing to the levels of these fatty acids could occur during IVM. However, the exact roles and biological significance of each specific lipid species in COCs maturation or their ability to develop remain largely unknown.

Furthermore, certain lipid synthesis and accumulation genes were increased in in vitro-matured cumulus cells, demonstrating that these pathways were upregulated 


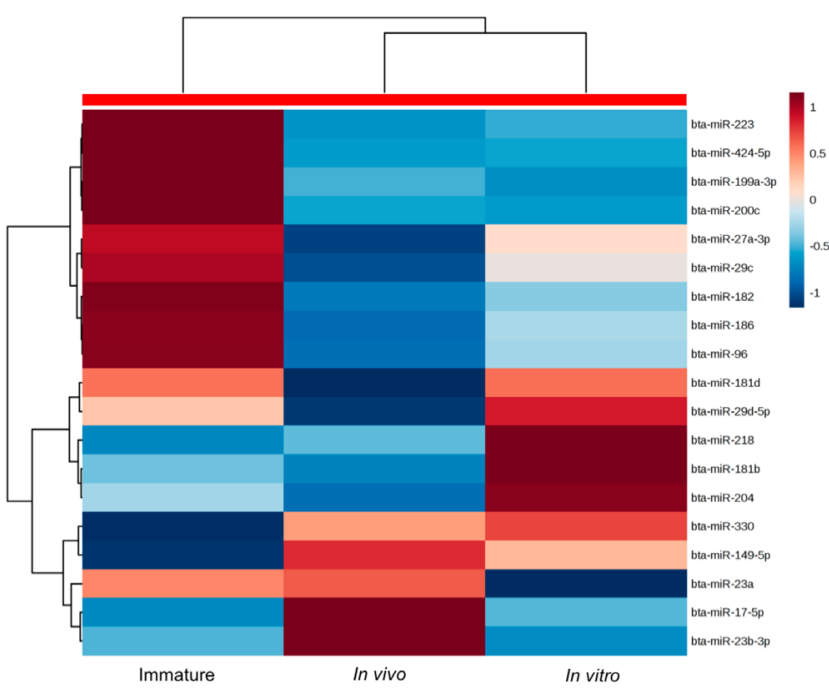

Figure 7 Hierarchical clustering and heat map depicting the expression levels of miRNAs differentially expressed among immature, in vivo-matured, and in vitro-matured cumulus cells.

compared to cumulus cells from in vivo-matured COCs. Our data confirmed our hypothesis that, during IVM, lipid synthesis occurred in cumulus cells. Additionally, fatty acid desaturation and elongation genes were also influenced, and these results were confirmed by MS analysis, from which we observed that the majority of the long-chain desaturated fatty acids were identified as increased in cumulus cells after in vitro culture.

Furthermore, our results suggest possible effects in mitochondrial activity and energy metabolism. The expression of $\beta$-oxidation and glycolysis-related genes in cumulus cells and ATP/ADP ratio in oocytes are indicative of reduced $\beta$-oxidation and increased glycolysis during IVM within COC, whereas the results of the ATP/ADP ratio demonstrated an alteration in energy demand in in vitro oocytes when compared to their in vivo counterparts. We note, however, that a wide spread of values was shown in in vivo-matured oocytes. Conversely, the higher similarity among in vitro samples, not observed in the in vivo group, likely occurred owing to the more similar conditions imposed to COCs during grouped in vitro culture. In addition to our ATP/ ADP results in oocytes, cumulus cell gene expression data were in accordance with this metabolic behavior, wherein the glucose metabolism genes G6PD, PFKP and $P G K 1$ were increased and the most highly expressed $\beta$-oxidation CPT1 gene, CPT1A, was decreased in the in vitro groups. An increase of the glycolysis pathway during IVM therefore may be a consequence of $\beta$-oxidation deficiency, providing an alternative form of energy supply. However, differences in glucose metabolism between in vivo- and in vitro-matured oocytes and cumulus cells are yet to be demonstrated.

In particular, the differential behavior of CPT1A and $C P T 1 B$ transcript levels between in vivo and in vitro systems potentially indicates that pre-transcriptional factors may influence these genes during IVM. However, we did not observe differences in DNA methylation as assessed by bisulfite DNA-sequencing analysis of the CPT1A promoter region of in vitro- and in vivo-matured cumulus cells (detailed in Supplementary data 1), suggesting that other mechanisms are likely involved in the transcriptional regulation of this gene.

Therefore, alterations in energy metabolism pathways identified in this study could, in part, be involved in lipid accumulation during IVM. Other authors have demonstrated that in vivo-matured oocytes showed higher mitochondria activity (Zeng et al. 2014) and lower $\beta$-oxidation levels when compared to in vivo-matured ones (Dunning et al. 2010, 2014). Altogether, these results suggest that there is a decrease in $\beta$-oxidation during IVM, which could lead to an increase of lipids in COCs.

Considering that our results and the literature suggest an increase in mitochondria activity in the in vivomatured oocytes, the increase of ROS levels observed in this group was expected. However, the amount of GSH, which constitutes the most abundant antioxidant in oocytes and embryos, drastically increased during in vivo maturation when compared with IVM. Oocytes must establish the GSH pool during maturation because, after this stage, its synthesis is halted until subsequent activation of the embryo genome occurs (EichenlaubRitter et al. 2011). This observation suggests an increased susceptibility to oxidative damage during in vitro embryo development.

Cumulus cells subjected to in vitro processes showed increased $B A X, B C L 2$ and CASP3 levels when compared to in vivo maturation, indicating an upregulation of apoptosis pathways. Additionally, although we detected higher levels of SOD1 in in vitro-matured cumulus cells, the levels of NRF2 and PRDX1 were lower compared to the in vivo process. Specifically, the NRF2 transcription factor has been demonstrated to be crucial for activating the most important antioxidant pathway and is associated with survival and the developmental competence of embryos cultured under conditions of oxidative stress (Amin et al. 2014). Therefore, our results indicate that in vitro culture leads to cellular stress without enhancing the antioxidant pathway. These findings are in accordance with those observed by Tesfaye and coworkers who showed an increase in cellular stress-related genes in cumulus cells retrieved from an IVM system (Tesfaye et al. 2009).

To the best of our knowledge, this is the first study evaluating metabolism and stress-related miRNAs during in vivo and in vitro maturation. As only six out of 88 miRNAs investigated varied during maturation and only two, bta-miR-17-5p and bta-miR-106b, differed between in vivo- and in vitro-matured oocytes, the posttranscriptional regulation of these pathway by miRNAs did not appear to constitute the driving mechanism leading to the observed differences in oocyte metabolism. 
The expression of mRNAs and miRNAs in cumulus cells showed both direct and indirect regulation of lipogenesis, with several components of these pathways varying between in vivo- and in vitro-derived COCs. In particular, the fatty acid elongation genes ACSL3 and ACSL6 exhibited different transcript levels after maturation. The miRNA-200c is predicted to regulate JUN, which leads to increased SREBP1 levels (Guo et al. 2016). Decreased expression levels of miR-200c in in vitro-matured cumulus cells may therefore lead to an increase in SREBP1 and, consequently, FASN and TG levels. Furthermore, miR-182 and miR-96, which were decreased in in vitro-matured cumulus cells, are predicted to regulate FBW7 and INSIG2, thereby negatively regulating SREBP1 synthesis (Jeon et al. 2013). Although these mechanisms have been described in humans and mice, they may also be conserved in bovines according to TargetScan. Therefore, changes in the levels of miR-200c, miR-182 and miR-96 in the same cells may control SREBP1 synthesis, which in our study was increased in in vitro cumulus cells.

Our gene expression results during in vivo and in vitro maturation paralleled those in the literature. Despite major gene expression differences observed during embryonic gene activation between 4- and 8-cell embryos (Jiang et al. 2014), several authors have observed a difference in gene expression between oocytes from in vitro and in vivo maturation processes (Kues et al. 2008, Katz-Jaffe et al. 2009). Moreover, in in vitro-matured oocytes, genes related to the tricarboxylic acid cycle and oxidative phosphorylation were downregulated when compared to their expression in in vivo-matured oocytes, indicating a metabolic disruption during IVM (Katz-Jaffe et al. 2009). Moreover, as mentioned previously, Tesfaye and coworkers observed an increase of cellular stress genes in in vitro-matured cumulus cells (Tesfaye et al. 2009). However, we could not discard the possibility that superovulation treatment in in vivo samples may have interfered in some results. Several authors have shown that FSH treatment during superovulation influences miRNA expression (Noferesti et al. 2013) and gene expression (Chu et al. 2012, Fortier et al. 2014). Nevertheless, until now, an interference in metabolism and cellular stress gene expression after FSH treatment has not been described. Altogether, our work and the literature data confirm that in vitro culture alters metabolism and cellular stress pathways in COCs.

Lipid accumulation, primarily of long-chain fatty acids such as palmitate, causes detrimental effects on mitochondria and ER leading to ER stress and apoptosis (Borradaile et al. 2006, Diakogiannaki et al. 2008). Moreover, it has been showed that bovine, mouse and human oocytes with excessive lipid accumulation exhibit increased levels of ER stress markers (Wu et al. 2010, Yang et al. 2012). As we showed that IVM can lead to increased lipid quantities in COCs and that these fatty acids primarily consist of a long-chain fatty acid, we may expect higher levels of stress in these cells. The results observed in the present study support that an increase in lipid accumulation consequent to the IVM system and a decrease of $\beta$-oxidation, combined with in vitro oxidative stress, may lead to the induction of ER stress and apoptosis.

Based on the negative relationship between lipids quantities, cell stress and apoptosis, it is important to understand the mechanisms involved in lipid accumulation during IVM. We hypothesized three possible pathways for lipid accumulation in the in vitro system: (1) an increase in lipids synthesis; (2) failure of $\beta$-oxidation and (3) fatty acid incorporation into oocytes. Here, we demonstrated a possibility of an increase of lipid synthesis and decrease of $\beta$-oxidation during IVM. Moreover, it was demonstrated that, depending on the type of supplementation (FBS or BSA), lipid accumulation occurred at different levels (Del Collado et al. 2016). We showed that the increased lipid accumulation in COCs is associated with a massive deregulation of lipid metabolism pathways in in vitro-matured cumulus cells, with no corresponding alterations in the gene expression profiles of the oocytes. Therefore, based on these results, it is tempting to speculate the existence of lipids trafficking to the oocyte cytoplasm, which may originate from the cell culture medium or from cumulus cells. We recently described potential fatty acid trafficking from cumulus cells to an oocyte via fatty acid-binding protein 3 and trans-zonal projections during oocyte maturation. This mechanism of lipid accumulation in oocytes is possibly more active during IVM when compared to in vivo maturation (Del Collado et al. 2017).

Thus, we demonstrated that IVM provided metabolically disrupted and stressed COCs. Furthermore, the in vitro system caused an increase in lipid synthesis and accumulation, modifications in the mono-, di- and triacylglycerols profiles, and an increase in cellular stress. Our results also suggest that IVM could affect mitochondrial activity, $\beta$-oxidation and glycolysis pathways during IVM, although more in-depth studies are needed to confirm this hypothesis. The mechanisms leading to these final cellular events are summarized in Fig. 8. In conclusion, our study provides evidence of metabolism and stress-related modifications during IVM of bovine oocytes. The results presented here encompass differences between in vivo and in vitro-matured COCs that may be attributed to the hormonal treatment prior to OPU, by the presence of serum in IVM or by several other potential factors influencing in vivo or in vitro maturation. The specific contribution of each of these factors is yet to be ascertained. These results highlight the necessity for further studies regarding the elucidation of cumulus cell-oocyte communication in order to better understand the underlying mechanisms involved in the poor quality of in vitro-produced oocytes and embryos. 

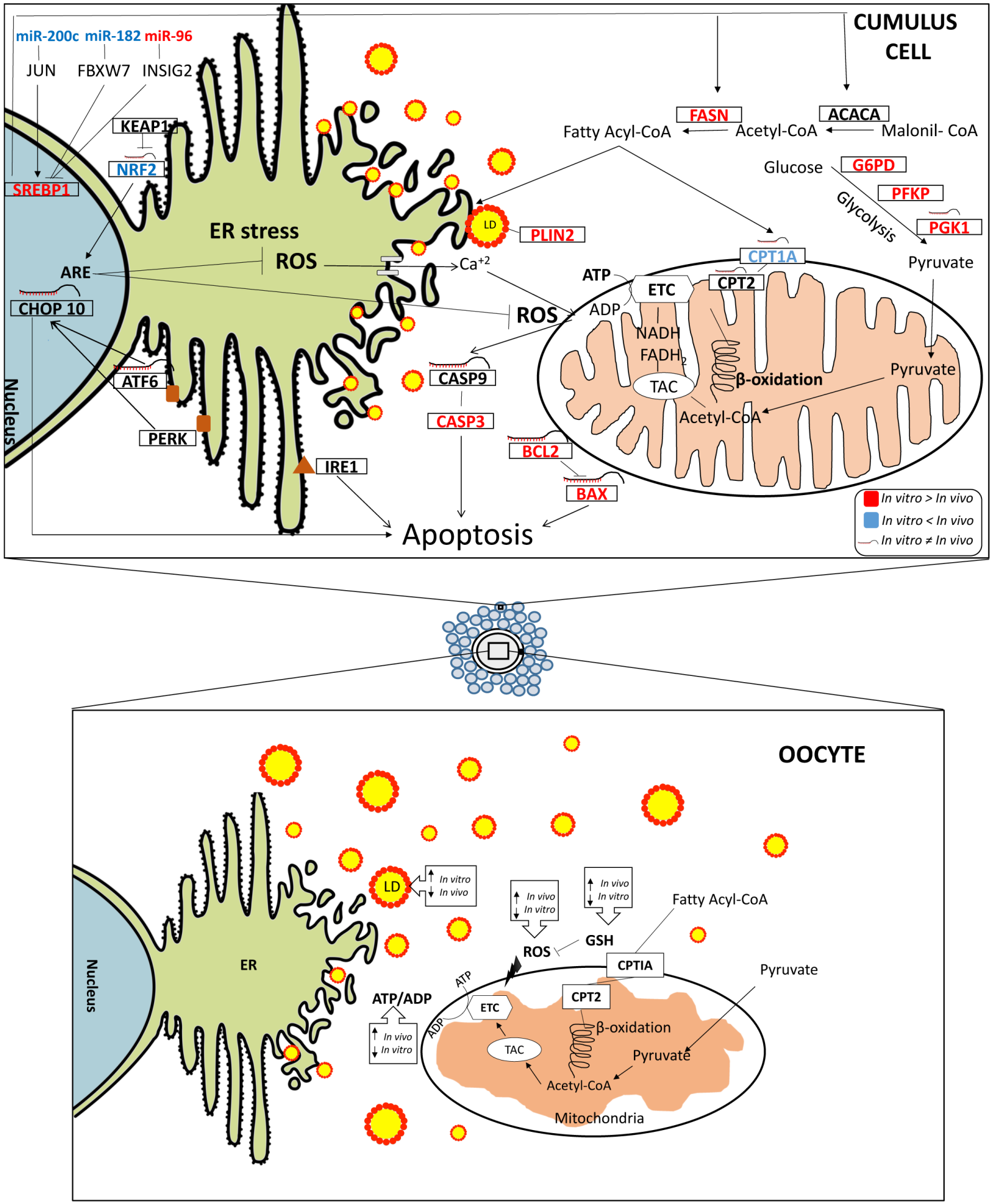

Figure 8 Summary of the modifications caused by the in vitro system in metabolism and cellular stress in cumulus cells and oocytes. Herein, we summarize the most important results that support the hypothesis that in vitro maturation leads to increase of lipid synthesis and accumulation, causing cellular stress within cumulus-oocyte complexes: in cumulus cells, besides the increase of neutral lipids, the expression levels of mRNAs (SREBP1, FASN e PLIN2) and miRNAs (miR-96 and miR-182) suggested an increase of lipid synthesis and accumulation in in vitro maturated cumulus cells. Moreover, we showed the increase of stress-related genes (CASP3, BCL2 and BAX), the decrease of antioxidant pathway related gene (NRF2) and the increase of glycolysis-related genes (G6PD, PKFP and PKG1) in in vitro maturated cumulus cells when compared to in vivo maturated cumulus cells. In oocytes, we demonstrated the increase of lipids and ROS levels and the decrease of GSH levels and ATP/ADP ratio in in vitro matured oocytes when compared to in vivo-matured oocytes. LD, lipid droplet; TAC, tricarboxylic acid cycle; ETC, electron transfer chain; ARE, antioxidant response element; ER, endoplasmic reticulum; ROS, reactive oxygen species. 


\section{Supplementary data}

This is linked to the online version of the paper at http://dx.doi.org/10.1530/REP-17-0134.

\section{Declaration of interest}

The authors declare that there is no conflict of interest that could be perceived as prejudicing the impartiality of the research reported.

\section{Funding}

This study was supported by the Sao Paulo Research Foundation - FAPESP (Grants 2014/21034-3, 2014/03281-3, 2014/22887-0, 2013/08135-2 and 2012/50533-2) and the National Counsel of Technological and Scientific Development (CNPq - grant 306978/2014-8).

\section{Acknowledgments}

The authors would like to thank the staff and students at the LMMD, Marcos Chiaratti, Gustavo Duarte, Marcel Nakashima, Hélio Alves Martins Júnior, José Luis Paz Jara, Patricia Kubo Fontes and Augusto de Castro Netto for their assistance with the sample collections, laboratory procedures and helpful discussions.

\section{References}

Aardema H, Vos PL, Lolicato F, Roelen BA, Knijn HM, Vaandrager AB, Helms JB \& Gadella BM 2011 Oleic acid prevents detrimental effects of saturated fatty acids on bovine oocyte developmental competence. Biology of Reproduction 85 62-69. (doi:10.1095/biolreprod.110.088815)

Abe H, Yamashita S, Satoh T \& Hoshi H 2002 Accumulation of cytoplasmic lipid droplets in bovine embryos and cryotolerance of embryos developed in different culture systems using serum-free or serum-containing media. Molecular Reproduction and Development 61 57-66. (doi:10.1002/ mrd.1131)

Amin A, Gad A, Salilew-Wondim D, Prastowo S, Held E, Hoelker M, Rings F, Tholen E, Neuhoff C, Looft C et al. 2014 Bovine embryo survival under oxidative-stress conditions is associated with activity of the NRF2mediated oxidative-stress-response pathway. Molecular Reproduction and Development 81 497-513. (doi:10.1002/mrd.22316)

Borradaile NM, Han X, Harp JD, Gale SE, Ory DS \& Schaffer JE 2006 Disruption of endoplasmic reticulum structure and integrity in lipotoxic cell death. Journal of Lipid Research 47 2726-2737. (doi:10.1194/jlr. M600299-JLR200)

Chu T, Dufort I \& Sirard MA 2012 Effect of ovarian stimulation on oocyte gene expression in cattle. Theriogenology 77 1928-1938. (doi:10.1016/j. theriogenology.2012.01.015)

Del Collado M, Saraiva NZ, Lopes FL, Gaspar RC, Padilha LC, Costa RR, Rossi GF, Vantini R \& Garcia JM 2016 Influence of bovine serum albumin and fetal bovine serum supplementation during in vitro maturation on lipid and mitochondrial behaviour in oocytes and lipid accumulation in bovine embryos. Reproduction Fertility and Development 28 1721-1732. (doi:10.1071/RD15067)

Del Collado M, da Silveira JC, Sangalli JR, Andrade GM, Sousa LR, Silva LA, Meirelles FV \& Perecin F 2017 Fatty acid binding protein 3 and transzonal projections are involved in lipid accumulation during in vitro maturation of bovine oocytes. Scientific Reports 7 2645. (doi:10.1038/ s41598-017-02467-9)

Diakogiannaki E, Welters HJ \& Morgan NG 2008 Differential regulation of the endoplasmic reticulum stress response in pancreatic beta-cells exposed to long-chain saturated and monounsaturated fatty acids. Journal of Endocrinology 197 553-563. (doi:10.1677/JOE-08-0041)

Downs SM, Mosey JL \& Klinger J 2009 Fatty acid oxidation and meiotic resumption in mouse oocytes. Molecular Reproduction and Development 76 844-853. (doi:10.1002/mrd.21047)

Dunning KR, Cashman K, Russell DL, Thompson JG, Norman RJ \& Robker RL 2010 Beta-oxidation is essential for mouse oocyte developmental competence and early embryo development. Biology of Reproduction 83 909-918. (doi:10.1095/biolreprod.110.084145)

Dunning KR, Anastasi MR, Zhang VJ, Russell DL \& Robker RL 2014 Regulation of fatty acid oxidation in mouse cumulus-oocyte complexes during maturation and modulation by PPAR agonists. PLOS ONE 9 e87327. (doi:10.1371/journal.pone.0087327)

Duru N, Gernapudi R, Zhang Y, Yao Y, Lo PK, Wolfson B \& Zhou Q 2015 NRF2/miR-140 signaling confers radioprotection to human lung fibroblasts. Cancer Letters 369 184-191. (doi:10.1016/j. canlet.2015.08.011)

Eichenlaub-Ritter U, Wieczorek M, Lüke S \& Seidel T 2011 Age related changes in mitochondrial function and new approaches to study redox regulation in mammalian oocytes in response to age or maturation conditions. Mitochondrion 11 783-796. (doi:10.1016/j. mito.2010.08.011)

Ferguson EM \& Leese HJ 1999 Triglyceride content of bovine oocytes and early embryos. Journal of Reproduction and Fertility 116 373-378. (doi:10.1530/jrf.0.1160373)

Fortier AL, McGraw S, Lopes FL, Niles KM, Landry M \& Trasler JM 2014 Modulation of imprinted gene expression following superovulation. Molecular and Cellular Endocrinology 388 51-57. (doi:10.1016/j. mce.2014.03.003)

Goto Y, Noda Y, Mori T \& Nakano M 1993 Increased generation of reactive oxygen species in embryos cultured in vitro. Free Radical Biology and Medicine 15 69-75. (doi:10.1016/0891-5849(93)90126-F)

Guo J, Fang W, Sun L, Lu Y, Dou L, Huang X, Sun M, Pang C, Qu J, Liu G et al. 2016 Reduced miR-200b and miR-200c expression contributes to abnormal hepatic lipid accumulation by stimulating JUN expression and activating the transcription of srebp1. Oncotarget 7 36207-36219. (doi:10.18632/oncotarget.9183)

Hossain MM, Salilew-Wondim D, Schellander K \& Tesfaye D 2012 The role of microRNAs in mammalian oocytes and embryos. Animal Reproduction Science 134 36-44. (doi:10.1016/j.anireprosci.2012.08.009)

Jeon TI, Esquejo RM, Roqueta-Rivera M, Phelan PE, Moon YA, Govindarajan SS, Esau CC \& Osborne TF 2013 An SREBP-responsive microRNA operon contributes to a regulatory loop for intracellular lipid homeostasis. Cell Metabolism 18 51-61. (doi:10.1016/j. cmet.2013.06.010)

Jiang Z, Sun J, Dong H, Luo O, Zheng X, Obergfell C, Tang Y, Bi J, O'Neill R, Ruan Y et al. 2014 Transcriptional profiles of bovine in vivo preimplantation development. BMC Genomics 15 756. (doi:10.1186/14712164-15-756)

Katz-Jaffe MG, McCallie BR, Preis KA, Filipovits J \& Gardner DK 2009 Transcriptome analysis of in vivo and in vitro matured bovine MII oocytes. Theriogenology 71 939-946. (doi:10.1016/j. theriogenology.2008.10.024)

Khurana NK \& Niemann H 2000 Energy metabolism in preimplantation bovine embryos derived in vitro or in vivo. Biology of Reproduction 62 847-856. (doi:10.1095/biolreprod62.4.847)

Kim JY, Kinoshita M, Ohnishi M \& Fukui Y 2001 Lipid and fatty acid analysis of fresh and frozen-thawed immature and in vitro matured bovine oocytes. Reproduction 122 131-138. (doi:10.1530/rep.0.1220131)

Kues WA, Sudheer S, Herrmann D, Carnwath JW, Havlicek V, Besenfelder U, Lehrach H, Adjaye J \& Niemann H 2008 Genome-wide expression profiling reveals distinct clusters of transcriptional regulation during bovine preimplantation development in vivo. PNAS 105 19768-19773. (doi:10.1073/pnas.0805616105)

Leroy JL, Vanholder T, Mateusen B, Christophe A, Opsomer G, de Kruif A, , Genicot G \& Van Soom A 2005 Non-esterified fatty acids in follicular fluid of dairy cows and their effect on developmental capacity of bovine oocytes in vitro. Reproduction 130 485-495. (doi:10.1530/ rep.1.00735)

Magenta A, Dellambra E, Ciarapica R \& Capogrossi MC 2016 Oxidative stress, microRNAs and cytosolic calcium homeostasis. Cell Calcium $\mathbf{6 0}$ 207-217. (doi:10.1016/j.ceca.2016.04.002) 
Marei WF \& Wathes DCFouladi-Nashta AA \& 2009 The Effect of Linolenic Acid on Bovine Oocyte Maturation and Development. Biology of Reproduction 81 1064-1072. (doi:10.1095/biolreprod.109.076851)

MárquezRuiz G, Jorge N, Martin-Polvillo M \& Dobarganes MC 1996 Rapid, quantitative determination of polar compounds in fats and oils by solid-phase extraction and size-exclusion chromatography using monostearin as internal standard. Journal of Chromatography $A \mathbf{7 4 9}$ 55-60. (doi:10.1016/0021-9673(96)00429-3)

McKeegan PJ \& Sturmey RG 2011 The role of fatty acids in oocyte and early embryo development. Reproduction Fertility and Development 24 59-67. (doi:10.1071/RD11907)

Miles JR, McDaneld TG, Wiedmann RT, Cushman RA, Echternkamp SE, Vallet JL \& Smith TPL 2012 MicroRNA expression profile in bovine cumulus-oocyte complexes: possible role of let-7 and miR-106a in the development of bovine oocytes. Animal Reproduction Science 130 16-26. (doi:10.1016/j.anireprosci.2011.12.021)

Noferesti SS, Hoelker M, Sohel MH, Salilew-Wondim D, Rings F, Schellander K \& Tesfaye D 2013 Effect of ovarian hyperstimulation on circulatory microrna profile in bovine follicular fluid and blood plasma. Reproduction Fertility and Development 25 251. (doi:10.1071/ RDv25n1Ab206)

Paczkowski M, Silva E, Schoolcraft WB \& Krisher RL 2013 Comparative importance of fatty acid beta-oxidation to nuclear maturation, gene expression, and glucose metabolism in mouse, bovine, and porcine cumulus oocyte complexes. Biology of Reproduction 88111. (doi:10.1095/biolreprod.113.108548)

Rizos D, Fair T, Papadopoulos S, Boland MP \& Lonergan P 2002 Developmental, qualitative, and ultrastructural differences between ovine and bovine embryos produced in vivo or in vitro. Molecular Reproduction and Development 62 320-327. (doi:10.1002/ mrd.10138)

Seidel GE 2006 Modifying oocytes and embryos to improve their cryopreservation. Theriogenology $65 \quad 228-235 . \quad$ (doi:10.1016/j. theriogenology.2005.09.025)

Shi L, Liu S, Zhao W \& Shi J 2015 miR-483-5p and miR-486-5p are downregulated in cumulus cells of metaphase II oocytes from women with polycystic ovary syndrome. Reproduction Biomedicine Online 31 565-572. (doi:10.1016/j.rbmo.2015.06.023)
Tesfaye D, Ghanem N, Carter F, Fair T, Sirard MA, Hoelker M, Schellander K \& Lonergan P 2009 Gene expression profile of cumulus cells derived from cumulus-oocyte complexes matured either in vivo or in vitro. Reproduction Fertility and Development 21 451-461. (doi:10.1071/ RD08190)

Thompson JG, Brown HM \& Sutton-McDowall ML 2015 Measuring embryo metabolism to predict embryo quality. Reproduction Fertility and Development 28 41-50. (doi:10.1071/RD15340)

Wright RW \& Ellington J 1995 Morphological and physiological differences between in-vivo-produced and in-vitro-produced preimplantation embryos from livestock species. Theriogenology 44 1167-1189. (doi:10.1016/0093-691X(95)00327-5)

Wu LL, Dunning KR, Yang X, Russell DL, Lane M, Norman RJ \& Robker RL 2010 High-fat diet causes lipotoxicity responses in cumulus-oocyte complexes and decreased fertilization rates. Endocrinology $\mathbf{1 5 1}$ 5438-5445. (doi:10.1210/en.2010-0551)

Yang X, Wu LL, Chura LR, Liang XY, Lane M, Norman RJ \& Robker RL 2012 Exposure to lipid-rich follicular fluid is associated with endoplasmic reticulum stress and impaired oocyte maturation in cumulus-oocyte complexes. Fertility and Sterility 97 1438-1443. (doi:10.1016/j. fertnstert.2012.02.034)

Yang Z, Cappello T \& Wang L 2015 Emerging role of microRNAs in lipid metabolism. Acta Pharmacologica Sinica B 5 145-150. (doi:10.1016/j. apsb.2015.01.002)

Zeng HT, Richani D, Sutton-McDowall ML, Ren Z, Smitz JE, Stokes Y, Gilchrist RB \& Thompson JG 2014 Prematuration with cyclic adenosine monophosphate modulators alters cumulus cell and oocyte metabolism and enhances developmental competence of in vitromatured mouse oocytes. Biology of Reproduction 91 47. (doi:10.1095/ biolreprod.114.118471)

Received 6 March 2017

First decision 13 April 2017

Revised manuscript received 14 September 2017

Accepted 2 October 2017 